Research Article

\title{
Off-site airway management: life-saving or life- threatening? a review of 74 incidences
}

\begin{abstract}
Introduction: Anaesthesiologists are considered as airway management experts in medical practice. They are frequently called upon to manage the airway of the suddenly critically ill patients at places outside the operation theatre (OT).

Objectives: It is intended to share the events, difficulties faced and to assist the newer anaesthesiologists in maintaining airway of critical patients at venues other than OT.

Methods: This retrospective observational study is done on 74 off-theatre emergency airway management calls attended at Combined Military Hospital (CMH) Dhaka, Bangladesh within a period over 6 months.

Results: Over 50 to 80 year-old age-group of both sexes was predominant (27\%). Calls were mostly attended at Emergency \& Casualty (32.4\%) during indoor duties. Total offhour calls were $64.9 \%$ and least but most serious calls were at dawn (10.8\%). Emergency intubations were required for patients at or impending cardiorespiratory arrest $(19 \%)$ due to head injury (14\%), cerebrovascular accidents (11\%) and massive myocardial infarction ( $2 \%)$. Requirement of airway management during non-surgical/interventional and imaging procedures was $21 \%$. Observations revealed that more preparedness, availability of necessary armamentarium and efficient assistance are yet to develop at the vulnerable sites of the hospital to avoid a potentially disastrous emergency "Can't Intubate, Can't Ventilate" scenario.

Conclusion: The incharges concerned have to ensure the availability of properly equipped airway management tools and drugs in various wards and clinical procedural suites. Ward nurses and paramedics are to be made efficient enough and familiar with the management protocol by regular bedside hands-on training.
\end{abstract}

Volume 4 Issue I - 2016

\author{
Md Rabiul Alam,' Md Mozibul Hoque2 \\ 'Classified Anaesthesiologist, Department of Anaesthesia \& \\ Intensive Care, Bangladesh \\ ${ }^{2}$ Advisor in Anaesthesiology, Department of Anaesthesiology, \\ Bangladesh
}

\section{Correspondence: Md Rabiul Alam, Classified}

Anaesthesiologist, Department of Anaesthesia \& Intensive Care, Bangladesh,Tel 8801769000737, Email rabiuldr@gmail.com

Received: August 19,2015 | Published: January 07, 2016

\section{Introduction}

At extremely critical scenarios, anaesthesiologists are considered as airway management experts in medical profession. In addition to their anaesthesia practice, they are frequently called upon to manage the airway of the suddenly critically ill patients at places outside the operation theatre (OT). ${ }^{1}$ They are also often assigned to provide monitored anaesthesia care or conscious sedation during various medical interventional and radiological imaging procedures. The situations in those areas are obviously less well-controlled and unfamiliar to them than that of in the OT. Although the rescuing protocols are definitely lifesaving for the victims, but sometimes may become disastrous and life-threatening due to unpreparedness, equipment failure, poor assistance and ineffective over-try. ${ }^{2}$ So, quick assessment, right decisions and purposeful actions are to be taken very rapidly with accuracy in these scenarios.

\section{Materials \& methods}

This retrospective observational study was done on 74 events encountered by the author while attending the emergency calls for airway management of the critically ill patients outside the OT at CMH Dhaka over a period of 6 months. The venues were: Emergency \& Casualty, Medical and Surgical HDUs, during patients' transportation by ambulance, Radiology department, Endoscopy suites, Catheterisation Lab, Coronary Care Unit and different wards. The scenarios, difficulties faced, actions taken and recommendations are tabulated and narrated descriptively.

\section{Results}

The demography of the patients is shown in Table 1 . The age variation was from new-born to over 80 years of both sexes. Over 50 to 80 years old age-groups were predominant. Figure 1, Table 2 and Figure 2 are showing the preponderances of venues, timings of emergency calls and diseases of the critically ill patients respectively. Calls were mostly attended at the Emergency \& Casualty (32.4\%) while on duties. Office hour's (0800 AM - 1430 PM) calls were $35.1 \%$ and the total off-hours' calls were $64.9 \%$. Least but most serious calls were at dawn hours $(10.8 \%)$. Emergency intubations were required for patients at or impending cardiorespiratory arrest $(19 \%)$ due to head injury (14\%), cerebrovascular accidents (11\%) and massive myocardial infarction (2\%). Requirement of airway management during non-surgical/interventional and imaging procedures was $21 \%$. Difficulties faced, actions taken and recommendations are summarised and narrated in Table 3.

\section{Discussion}

At least one in four major airway events in a hospital are likely to occur outside the OT; i.e., in ICUs, Emergency \& Casualty department, HDUs and at other venues. ${ }^{3}$ The outcome of these events is particularly adverse. Analysis has identified repeated gaps in care are: delayed identification of the patient's at-risks, poor or incomplete planning, inadequacy of skilled staffs and equipment to manage these events successfully, and failed rescue due to lack of or failure of interpretation of capnography. ${ }^{4}$ 
Table I Demography of the critically ill patients for whom the emergency airway management calls were attended outside the operation theatres

\begin{tabular}{|c|c|c|c|c|c|}
\hline \multirow[t]{2}{*}{ Sex/Age } & \multicolumn{5}{|c|}{ Age Groups (Years) \& \% } \\
\hline & $0-1$ & 02-Dec & $13-50$ & $51-80$ & Over 80 \\
\hline Male $(n=40)(54 \%)$ & $4(5.4 \%)$ & $8(10.8 \%)$ & $6(8.1 \%)$ & $12(16.2 \%)$ & $10(13.5 \%)$ \\
\hline Female $(n=34)(45.9 \%)$ & $2(2.7 \%)$ & $10(13.5 \%)$ & $8(10.8 \%)$ & $8(10.8 \%)$ & $6(8.1 \%)$ \\
\hline Total $(n=74)$ & $6(8.1 \%)$ & $18(24.3 \%)$ & 14 (18.9) & $20(27.0 \%)$ & $16(21.6 \%)$ \\
\hline
\end{tabular}

Table 2 Timings of the Emergency Calls for Airway Managements

\begin{tabular}{llllll}
\hline Times & At dawn (5:00-7:00 & Office hours (8:00AM- Evening (2:3 I- & \multicolumn{2}{l}{ Midnight (I2:00PM- } & Late night (2:00- \\
& AM) & 2:30PM) & I I:00PM) & 2:00AM) & 5:00AM) \\
\hline Calls & 8 & 25 & 19 & 12 & 10 \\
$(n=74)$ & $-10.80 \%$ & $-35.10 \%$ & $-24.30 \%$ & $-16.20 \%$ & $-13.50 \%$ \\
\hline
\end{tabular}

Table 3 Situations/Difficulties faced, actions taken while attending the calls for emergency airway management outside the operation theatres and recommendations

Situations/Difficulties faced
Rescuers are more busy with ventilation rather
than effective chest compressions in case of cardiac
arrest victims
Stomach of patient is distended with gas due to
improper/over-enthusiastic bag-mask ventilation/
without using airway

Intubation trolley and required drugs are not ready properly

Plates of the beds at the head-ends are not negotiable

Suction facilities on the spot is not available or not functioning properly

Unavailability of different sizes of ETT, stylet; larygoscopes light failure, cuff-leakage, lack of efficient assistance are not very uncommon

\section{Actions taken}

Appropriate importance given

simultaneously to vent
chest compressions

Correct AMBU support given using appropriate airway. N/G tube inserted immediately where possible

Tried to manage those instantaneously Adopted the improvised position of the patient with difficulties

Arranged quickly and managed

Tried to manage, used the alternatives with difficulties

\section{Recommendations}

Recent and updated protocols for CPR

(C-A-B) to be trained and practiced regularly by the health caregivers

Ward nurses and paramedics are to be trained and familiar on proper procedures of bag-mask ventilations using appropriate sized airway

Everyday regular morning check is to be practiced

Provision for detachment of head-end plate of all hospital-beds is required

Regular check-up and maintenance is necessary

Regular morning check-up drill in every wards has no alternatives

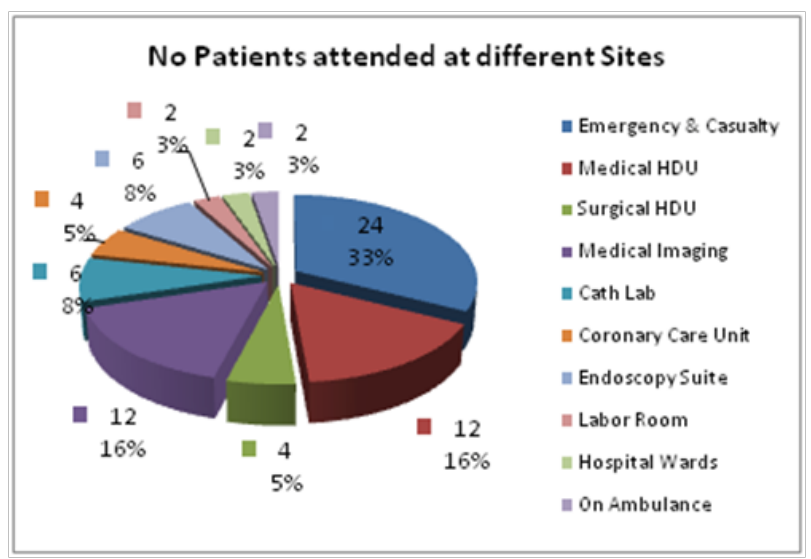

Figure I Pie-chart showing the number \& percentages of patients attended at different sites. Note: Data of Critical Care Centre is excluded.

Airway management outside the OT may be required for:

A. Moribund patients who are deeply obtunded require rapid intubation with minimal assessment and no requirement for sedation or muscle relaxation.

B. Semi-elective intubation is done to those patients who often have neuromuscular or CNS pathology which is slowly deteriorating, but has no cardio respiratory compromise. A full airway assessment and routine intubation, similar to that performed in the OT may be carried out.
C. Emergency intubation requires to the patients having a variety of pathologies including:

a. Airway obstruction from tumour, trauma or infection.

b. Primary pulmonary pathology including: pneumonia, ARDS and lung contusions.

c. Multiple organ failure with pulmonary oedema.

d. Severe CNS trauma including: head injury, subarachnoid haemorrhage and stroke leading to progressive central hypoventilation and airway protection failure. ${ }^{5}$

Airway assessment protocol and difficult airway algorithm have been formalized by the American Society of Anesthesiologists (ASA), which is revised periodically. ${ }^{6}$ The basis of the assessment is evaluation of factors that may hinder the displacement of the soft tissues of the mouth and neck and thus obscure the laryngoscopic view of airway. The full assessment requires evaluation of teeth, mouth opening, jaw, neck movement and deformities, but should take less than 2 minutes with regular practice. Approaches after assessment ${ }^{7}$ are:

a. Rapid sequence induction: If no difficulties are anticipated, sedation and muscle relaxation may be used.

b. Sedation only: With 1-2 difficulties anticipated, only sedatives may be used, but muscle relaxants are to be avoided.

c. A wake intubation/tracheostomy: If all conventional manoeuvre and interventions deemed difficult, the airway should be secured awake. 


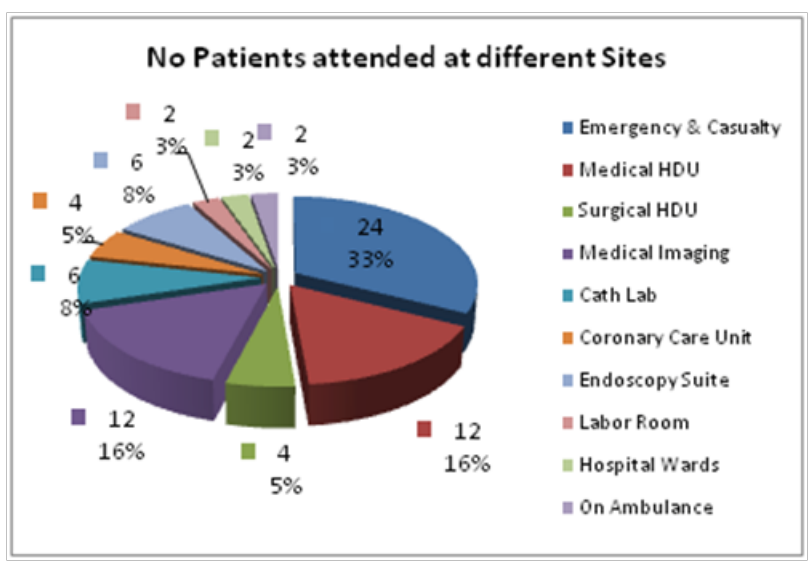

Figure 2 Doughnut-chart showing the diseases of the patients attended for emergency airway management.

In a hospital environment, all clinical nursing areas should have a resuscitation trolley consisting of the equipment for emergency airway management. The list of necessary equipment may be as in the Oxford Airway Box ${ }^{8}$ as following: suture materials, needles, cannulas (peripheral and central), syringes, gauzes, swabs, scalpel, dressing pack (galipot, drapes, needle holder, forceps), lubricants, gum elastic bougie, pen torch/headlight, suction catheters, Yankauer sucker, oro- and nasopharyngeal airways, varieties of ETTs with stylets and LMAs, percutaneous tracheostomy set, cricothyrotomy kit, AMBU bag capable of delivering 100\%oxygen, functioning laryngoscope with a variety of blades, and drugs including adrenaline, lignocaine, atropine, glycopyrrolate, sedatives and musclerelaxants. In an urgent, rather than an emergency situation, a fibreoptic bronchoscope (FOB) may be obtained.

An adequate IV access is to be established first, as hypotension is a common consequence following intubation and inotropes may require. Measures to prevent aspiration is obligatory. In case of difficulties or failing, persistent attempts of laryngoscopy should be avoided. Intermittent oxygenation by a facemask with continued application ofcricoid pressure should be performed to prevent significant desaturation $(<95 \%)$. Visualization of the larynx may be improved by changing the size and type (Macintosh to Miller) of laryngoscope blade and externally manipulating the larynx using the BURP (backward, upward, rightward pressure) manoeuvre. ${ }^{9}$

The most certain confirmatory test of successful endotracheal intubation is visualization of the tube as it passes through the vocal cords. Tube misting and auscultation may be useful but are not reliable. ${ }^{10}$ Visualization may not always be possible and other confirmatory tests have been employed. The most widely used tool in the OT is capnography, but unfortunately this facility may not be available at the off-sites. Other method is visualization of tracheal rings and carina using an FOB, but this scope may not be readily available. ${ }^{11}$ Both the cuff palpation and trans-illumination with a light wand have been used, but neither is superior to auscultation. Auscultation is substantially inferior tocapnography. ${ }^{12}$ Direct visualization with a laryngoscope or FOB thus remains the gold standard of confirmation.

\section{Conclusion}

Emergency airway management at any site is lifesaving for the moribund patients in clinical practice. Although the consequences are not so optimistic with pre-existing irreversible massive organ damage instead of following all prescribed protocols meticulously. But, the rescue measures unfortunately may become sometimes lifethreatening due to improper assessment, actions and futile over-try in difficult scenarios. Moreover, off-theatre circumstances are often revealed quite odd and unfamiliar to the caregivers. Therefore, proactive preparedness, early detection and referral, correct decisions, purposeful actions and trained assistance are solicited for better outcomes.

\section{References}

1. Pugh H, Le S, Mclennan J. A review of pre-admission advanced airway management in combat casualties, Helmand Province 2013. J R Army Med Corps. 2015;161(2):121-126.

2. Cook T, Behringer E, Benger J. Airway management outside the operating room: hazardous and incompletely studied. Curr Opin Anaesthesiol. 2012; 25(4):461-469.

3. Cook T, Woodall N, Frerk C. Major complications of airway management in the UK: results of the Fourth National Audit Project of the Royal College of Anaesthetists and the Difficult Airway Society. BJA. 2011;106(5):632-642.

4. Astin J, Cook TM, King EC, et al. Timely safe airway management in critically ill patients. BJA. 2013;110(2):315-316.

5. Hodgson E. Airway management outside the operating theatre. SJA. 2008;2(2):35-39.

6. Practice guidelines for management of the difficult airway: an updated report by the American Society of Anesthesiologists Task Force on management of the difficult airway (2003). Anesthesiology. 2003;98(5):1269-1277.

7. Nee PA, Benger J, Walls RM. Airway management. Emerg Med J. 2008;25:98-102.

8. Banga R, Thirlwall A, Corbridge R. How Well Equipped are ENT Wards for Airway Emergencies? Ann R Coll Surg Engl. 2006;88(2):157-160.

9. Takahata O, Kubota M, Mamiy K, et al. The efficacy of the "BURP" maneuver during a difficult laryngoscopy. Anesth Analg. 1997;84(2):419-421.

10. Salem MR. Verification of endotracheal tube position. Anesthesiol Clin North America. 2001;19(4):813-839.

11. Weiss YG, Deutschman CS. The role of FOB in airway management of the critically ill patient. Crit Care Clin. 2000;16(3):445-451.

12. Resuscitation Council UK Guidelines (2010). Accessed in May 2015 from www.resus.org.uk. 